\title{
Impact of Hepatitis C Virus Infection and Direct-acting Oral Antiviral Drugs on Glycaemic State in Type 2 Diabetic Patients
}

\author{
Ayman ElBadawy, Mohamed El sayed, Islam Abdellatif, Rasha AbdelMoneim,
}

\begin{abstract}
a Department of internal

Background: Diabetes mellitus (DM) is strongly associated with medicine, Benha faculty of medicine, Benha University, Egypt. chronic hepatitis $\mathrm{C}$ virus (HCV) infection. This work aimed to estimate the impact of Hepatitis $\mathrm{C}$ virus infection on glycaemic state and insulin resistance in type 2 Diabetic patients, and evaluate the effect of direct-

Correspondence to: Islam acting oral antiviral treatment on the glycaemic state and insulin Abdellatif, Department of internal medicine, Benha faculty of medicine, Benha University, Egypt.

Email:

islambenha2021@gmail.com

Received: 14 January 2021

Accepted: 22 February 2021 resistance in type 2 Diabetic patients. Methods: The study was performed on (40 patients) with Type 2 Diabetes who were infected by Hepatitis C Virus and took Direct-acting Oral Antiviral Drugs. The study also included (10 patients) who had Type 2 Diabetes and HCV infection serving as control group. All patients and control were subjected to thorough history taking, clinical examination, and lab. investigations as FBG, PP and HBA1c, Serum fasting Insulin level, And HOMA-IR, HCVAb - HBsAg - HIVAb, CBC, SGOT, SGPT, Serum Albumin and Bilirubin, PCR for HCV RNA, and Abdominal US. Results: DAAs-based eradication of $\mathrm{HCV}$ is associated with improved glycemic control in patients with type 2 diabetes as evidenced by a significant reduction of mean FBG, Fasting insulin, HbA1c, and HOMA IR. There was a positive correlation between the treatment of $\mathrm{HCV}$ infection and the reduction of anti-diabetic drugs used in the studied patients. Conclusion: DAAs-based eradication of $\mathrm{HCV}$ is associated with improved glycemic control in patients with type 2 diabetes.
\end{abstract}

Keywords: HCV; DAAs; Type 2 DM 


\section{Introduction}

Hepatitis $\mathrm{C}$ virus (HCV) infection is the major cause of cirrhosis and hepatocellular carcinoma worldwide. $\mathrm{HCV}$ is a diabetogenic pathogen in which glucose irregularities are common in patients with chronic HCV infection (CHC). There is strong scientific evidence showing the prominent association between $\mathrm{CHC}$ and type-2 diabetes mellitus (1).

In patients without known DM, there was a 3.5 -fold increase in the frequency of glucose irregularities in $\mathrm{CHC}$ patients in contrast with controls. Insulin resistance (IR) impaired treatment efficacy of PEGylated interferon and ribavirin therapy, while successful eradication of $\mathrm{HCV}$ improved IR and glucose irregularities in CHC patients (2).

Meanwhile, clearance of HCV improved betacell function and hepatic expression of insulin receptor substrate-1 and -2 , the central molecules of the insulin signaling cascade. IR and systemic inflammation could therefore be enhanced by direct acting antiviral therapy (3).

The liver has an important role in carbohydrates metabolism since it is responsible for the balance of blood glucose levels by means of glycogenolysis and glyconeogenesis. In the presence of hepatic disease, the metabolic homeostasis of glucose is impaired as a result of disorders such as insulin resistance ,glucose intolerance and diabetes (4).

The association between liver cirrhosis and DM was first described at 1976 (5) in which $57 \%$ of cirrhotic patients showed increased insulin resistance. Insulin resistance has consistently been found to be closely linked to fibrosis in $\mathrm{HCV}$ infection, although typically associated with T2DM in prefibrotic stages. $\mathrm{HCV}$ infection could be associated with a reduced prevalence of metabolic syndrome owing to virus-associated reduction in Body Mass Index and hypobetalipoproteinemia (6).

This could be due to higher serum insulin levels in diabetic patients with chronic liver disease than those in patients with life-style related diabetes, suggesting that besides over-eating, obesity and physical inactivity, other distinctive factors may underlie the pathophysiology of hyperinsulinemia in patients with chronic liver disease (7).

Only 20 years after the discovery of the Hepatitis C Virus (HCV), a cure is now likely for most people affected by this chronic infection, which carries a substantial disease burden. The recent approval of two direct- 
acting antiviral agents that specifically inhibit viral replication has dramatically increased the viral clearance rate, from less than $10 \%$ with the initial regimen of interferon monotherapy to more than $70 \%$ with current therapy (8).

Currently, direct-acting antiviral drugs (DAA) are the gold standard for treating $\mathrm{HCV}$ infection, while yielding SVR in nearly all patients. In chronic HCV patients with T2DM, DAA therapy is associated with both better fasting glucose and glycated hemoglobin (HbA1C) controls; thus reducing pharmacotherapy in a certain part of patients is possible (9).

In other studies; in course of DAA drug therapy, Six DAA regimens showed high sustained virological response (SVR) rates $(>95 \%)$ in patients with $\mathrm{HCV}$ genotype 1 infection without cirrhosis. Patients with hepatic decompensation, particularly those with Child-Turcotte-Pugh class C disease, had lower SVR rates (78\% to $87 \%$ ) than other populations. The addition of ribavirin was associated with increased SVR rates for certain DAA regimens and patient groups. Overall rates of serious adverse events and treatment discontinuation were low $(<10 \%$ in the general population); regimens that included ribavirin had more mild or moderate adverse events than those without (10).
Moreover, DAA treatment of chronic hepatitis C improves hypertension control and atherosclerotic plaques. It is very likely that DAA therapeutic regimens will decrease both T2DM prevalence and cardiovascular risk in chronic hepatitis $\mathrm{C}$ patients; further research, however, is needed (11).

Patients with chronic hepatitis $\mathrm{C}$ have both higher prevalence of diabetes mellitus type 2 (T2DM) and increased cardiovascular risk compared to never infected people. More importantly, DAA therapy leads to both better fasting glucose and $\mathrm{HbA} 1 \mathrm{C}$ controls in patients with T2DM. Reducing antidiabetic treatment in some of the patients is possible (12).

The overcoming of IR as a host predictive factor for SVR appears to be one of the great advancements by DAAs. The post-treatment glucose homeostasis status in CHC patients is an intriguing issue for clarification due to the diabetogenic effect of HCV per se. Successful eradication of $\mathrm{HCV}$ after antiviral therapy prevents the development of de novo IR among those nondiabetic patients in the longterm fashion (13).

This work aimed to estimate the impact of Hepatitis C virus infection on glycaemic state and insulin resistance in type 2 Diabetic patients, and evaluate the effect of directacting oral antiviral treatment on the 
glycaemic state and insulin resistance in type 2 Diabetic patients.

\section{Patients and methods}

This is a prospective case-control study which was conducted on patients attending Benha University Hospital at Qalubiya governorate.

During the period from September 2019 to September 2020.

This study was approved by the Ethical Committee of Benha Faculty of Medicine. A written informed consent was taken from all patients included in the study.

The study was performed on (40 patients) with Type 2 Diabetes who were infected by Hepatitis C Virus and took Direct-acting Oral Antiviral Drugs. The study also included (10 patients) who had Type 2 Diabetes and HCV infection serving as control group.

All patients had been informed about the purpose of the study and signed consent before inclusion.

\section{Inclusion criteria:}

Patients with Type 2 Diabetes Mellitus infected by Hepatitis C Virus.

\section{Exclusion criteria:}

Patients with Type 1 Diabetes Mellitus

Patients with chronic liver diseases other than HCV infection as:
1. Hepatitis B Virus infection

2. Metabolic Liver Diseases (Hemochromatosis, Wilson's disease)

3. Bilharzial infection.

All patients and control were subjected to thorough history taking, clinical examination, and lab. investigations as FBG, PP and HBA1c, Serum fasting Insulin level, And HOMA-IR, HCVAb - HBsAg - HIVAb, CBC, SGOT, SGPT, Serum Albumin and Bilirubin, PCR for $\mathrm{HCV}$ RNA, and Abdominal US.

\section{Statistical analysis:}

All collected data will be tabulated and analyzed By ( SPSS) as a suitable statistical method and illustrated by diagrams .categorical data were presented as number and percentages while quantitative data were expressed as mean \pm standard deviation and range. Fisher's exact test(FET) was used to analyse categorical variables. Quantitative data were tested for normality at $\mathrm{p}$ value $>0.05$

\section{Results}

Patients had an age range between 41 and 76 years old with a mean of $55.82 \pm 8.968$ years. Regarding sex of the patients, 28 patients (56 $\%)$ were males and 22 (44\%) were females. The Body Mass Index ranged between 21.7 and 33.9 with a mean of $27.2 \pm 2.79$. It also 
shows that a total of 18 patients $(36 \%)$ were employers, while 16 patients (32\%) were farmers and 16 patients (32\%) were housewives. table 1

Types of Anti-diabetic Treatments among patients, shown in figure 1, and Types of Direct-acting Antiviral Regimens among the $\mathrm{HCV}$ patients, figure 2

Table 2 shows the difference between the responders (SVR) and the non-responders (No SVR) according to FBG, Fasting insulin, HbA1c and HOMA-IR in these patients before and after treatment with the mean change in their value. It also shows a highly significant $p$ value in the reduction of $\mathrm{FBG}, \mathrm{HbA1c}$ and HOMA-IR in these patients confirming the significance of treatment of $\mathrm{HCV}$ infection on the glycaemic control.

Table 3 shows the average number of antidiabetic drugs and the average dose of insulin used by these patients before and after treatment of $\mathrm{HCV}$ with DAA and the mean change in their value. It also shows a highly significant $\mathrm{p}$ value which means a positive correlation between the treatment of $\mathrm{HCV}$ infection and the reduction of anti-diabetic drugs used in these patients.

Table (1): Socio-demographic data of the studied group:

\begin{tabular}{ccc}
\hline Variables & Number & $\%$ \\
\hline Age $($ years $)$ & Mean \pm SD & $55.82 \pm 8.97$ \\
& Range & $41-76$ \\
BMI $\left(\mathbf{K g} / \mathbf{m}^{2}\right)$ & Mean \pm SD & $27.21 \pm 2.79$ \\
& Range & $21.7-33.9$ \\
Male & Sex & \\
Female & $\mathbf{2 8}$ & $56 \%$ \\
& $\mathbf{2 2}$ & $44 \%$ \\
Employer & Occupation & $36 \%$ \\
Farmer & $\mathbf{1 8}$ & $32 \%$ \\
Housewife & $\mathbf{1 6}$ & $32 \%$ \\
\hline
\end{tabular}


Table (2): Comparison between Responder (SVR) and Non-Responder (No SVR) patients regarding parameters of glycaemic control:

\begin{tabular}{lclllll}
\hline & & Pre-treatment & Post-treatment & $\begin{array}{c}\text { Mean } \\
\text { Change }\end{array}$ & $\begin{array}{c}\text { Paired t } \\
\text { test }\end{array}$ & \multicolumn{1}{c}{ P value } \\
\hline \multirow{2}{*}{ FBG } & SVR & $189.75 \pm 14.81$ & $168.50 \pm 13.26$ & 21.25 & 7.59 & $<0.001^{* *}$ \\
& No SVR & $208.16 \pm 30.84$ & $171.03 \pm 29.45$ & 37.13 & 23.72 & $<0.001^{* *}$ \\
Fasting & SVR & $17.75 \pm 1.67$ & $16.75 \pm 2.12$ & 1 & 3.06 & $0.018^{*}$ \\
insulin & No SVR & $17.01 \pm 1.85$ & $14.69 \pm 2.51$ & 2.32 & 10.08 & $<0.001^{* *}$ \\
& SVR & $7.38 \pm 1.06$ & $7.0 \pm 0.76$ & 0.38 & 2.05 & 0.08 \\
HbA1c & No SVR & $8.94 \pm 1.32$ & $7.97 \pm 1.23$ & 0.97 & 8.47 & $<0.001^{* *}$ \\
& SVR & $8.3 \pm 1.03$ & $7.01 \pm 1.11$ & 1.29 & 5.69 & $0.001^{* *}$ \\
HOMA-IR & No SVR & $8.76 \pm 1.41$ & $6.18 \pm 1.32$ & 2.58 & 18.86 & $<0.001^{* *}$ \\
& & & & & &
\end{tabular}

Table (3): Effect of HCV treatment on the use of anti-diabetic medications (treatment of Diabetes):

\begin{tabular}{cccccc}
\hline & Pre-treatment & Post-treatment & Mean Change & & \\
& $\begin{array}{c}\text { Mean } \pm \text { SD } \\
\text { Range }\end{array}$ & $\begin{array}{c}\text { Mean } \pm \text { SD } \\
\text { Range }\end{array}$ & $\begin{array}{c}\text { In } \\
\text { Value }\end{array}$ & Paired t test & P value \\
\hline $\begin{array}{c}\text { Average Number } \\
\text { of anti-diabetic }\end{array}$ & $2.65 \pm 0.46$ & $2.01 \pm 0.32$ & & & \\
drugs & $2-3$ & $2-3$ & 0.64 & 3.04 & 0.07 \\
& & & & \\
Oral anti-diabetic & $3.73 \pm 0.74$ & $2.25 \pm 0.46$ & 1.48 & 5.26 & $0.01^{*}$ \\
drugs (number) & $2-4$ & $2-4$ & & 12.46 & $<0.001^{* *}$ \\
$\begin{array}{c}\text { Average Dose of } \\
\text { Insulin (Units) }\end{array}$ & $62.5 \pm 7.67$ & $47.5 \pm 6.32$ & 15 & & \\
\hline
\end{tabular}




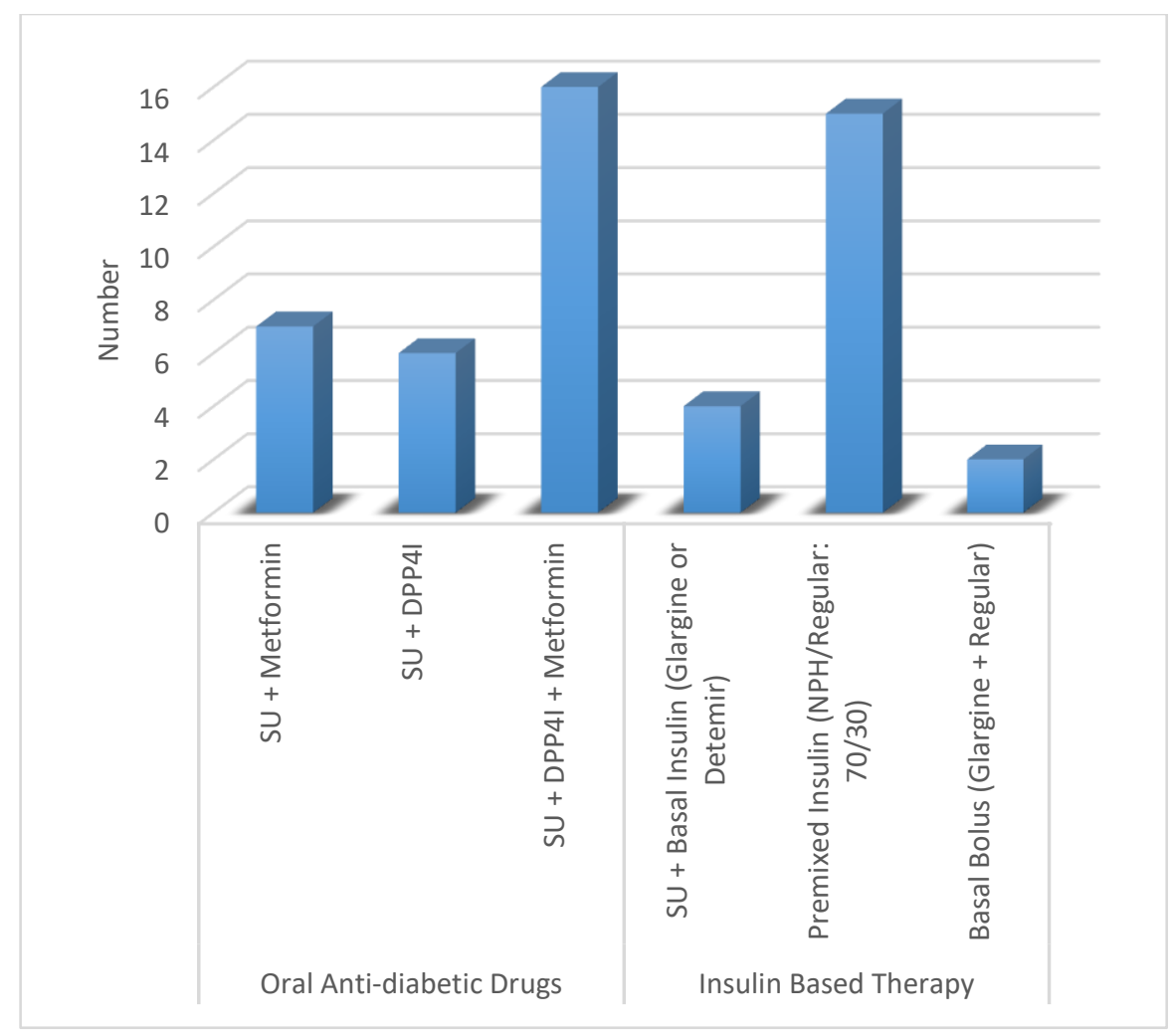

Figure (1): Types of Anti-diabetic Treatments among patients

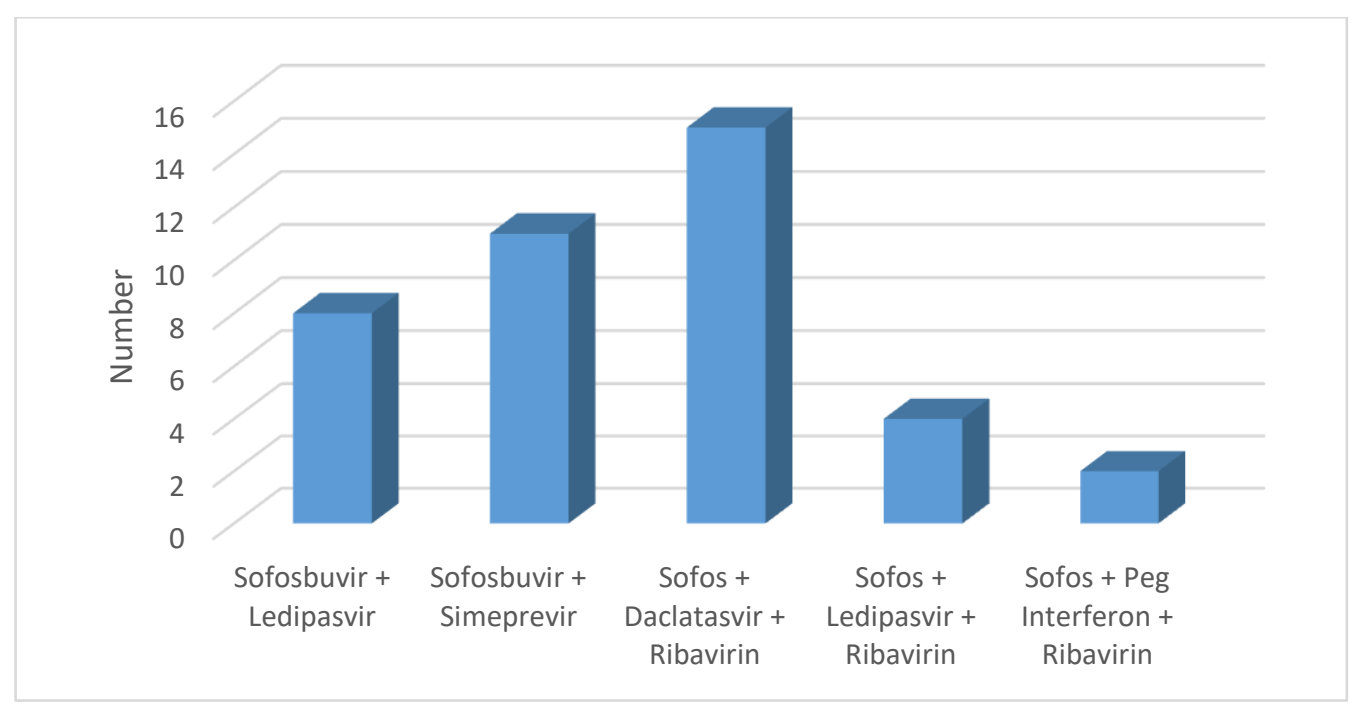

Figure (2): Types of Direct-acting Antiviral Regimens among the HCV patients: 


\section{Discussion}

In this study, we have assessed 50 diabetic patients who were infected with Hepatitis C Virus, for their blood glucose changes and glycemic parameters over the period of 12 weeks. Only $40(80 \%)$ of these patients took direct-acting antiviral drugs for 12 weeks and $36(72 \%)$ of them achieved SVR12 while the other $4(8 \%)$ did not. There were $10(20 \%)$ patients serving as control group.

In this study, the diabetic patients who achieved SVR12 have a higher average blood glucose and $\mathrm{HbA} 1 \mathrm{c}$ reduction after DAA therapy than those who did not achieve SVR12; (37.13 mg/dl, 0.97\%) and (21.25 $\mathrm{mg} / \mathrm{dl}, 0.38 \%$ ) respectively.

These results are in agreement with those reported at 2017 (14), were 55 (73.3\%) patients showed improvement of FBS, HOMA-IR, and HBA1c at the end of therapy, and improvement was significantly associated with younger age and low BMI. The improvement also related to less disease duration, low FBS, and negative family history. However, liver condition and viral load have no statistically significance, and this may be due to all patients in study being Child A.

The same was reported before (15) in which it was found that the drop in $\mathrm{HbAlc}$ associated with antiviral treatment was greater in those who achieved SVR $(0.98 \%)$ than in those who sustained treatment failure $(0.65 \%)$ (Adjusted mean difference 0.34, P = $0.02)$.

With similar results, in a study (16), they followed $122 \mathrm{HCV}$-positive patients with T2DM receiving DAAs and evaluated their FG and HbA1c levels at baseline and 12 weeks post-treatment; the influence of SVR on antidiabetic medication needs was also assessed. Potentially confounding factors affecting the results (i.e. weight variation) were considered. Based on a multivariable regression analysis, SVR was the only independent predictive factor associated with the improvement of glycaemic control $(\mathrm{OR}=$ 49.75, 95\% CI 2.197-112.67, $\mathrm{p}=0.014)$.

In a single case report, a significant improvement in diabetic control after successful HCV treatment with DAA therapy, was found. This improvement in insulin requirements and $\mathrm{HbA} 1 \mathrm{c}$ persisted following viral clearance despite an increase in the patient's BMI. Control of T2DM is usually advised prior to HCV therapy in order to increase response rates (17).

In this study, the diabetic patients who achieved SVR12 were having lower values of 
average HOMA IR $(6.18 \pm 1.32)$ than those who didn't $(7.01 \pm 1.11)$ after 3 months of treatment with direct-acting antiviral drugs.

Another study, (18) provided evidence showing that HCV-induced IR could be overcome by the potent DAAs within a short treatment period. It thus provided a concordant observation with the previous study showing the efficacy of the DAAs was not ameliorated by IR. The study demonstrated that pre-treatment IR had no significant impact on the treatment efficacy of DAAs. IR as well as BMI and HbA1c did not significantly change at EOF compared to their baseline values. However, we observed there was a significant improvement of betacell function at EOF, especially among those who had baseline high IR. The results from this preliminary study indicate that beta-cell function might be improved in the earliest manner during antiviral therapy.

Viral load assessment before, during, and after therapy is an important tool for predicting the treatment outcome. Although viral load does not correlate with the severity of liver injury or the progression of the disease, a low baseline viral load (<600 000$800000 \mathrm{IU} / \mathrm{ml}$ or less) is an independent predictor of SVR regardless of genotype in many studies, and patients with pre-treatment high viral loads have worse long-term outcomes than patients with low viral loads (14).

In this study, we find that the diabetic patients who achieved SVR12 had to reduce their antidiabetic medications $(30 \%$ reduction of oral drugs taken and $25 \%$ reduction of insulin dose), while the other patients who did not achieve SVR didn't need to reduce their dose of antidiabetic medications.

Use of antidiabetic medications decreased more in patients who achieved SVR than in those who sustained treatment failure, especially for the use of insulin, which dropped significantly from $41.3 \%$ to $38 \%$ in patients achieving SVR compared with a slight increase from $49.8 \%$ to $51 \%$ in those who sustained treatment failure (15).

\section{Conclusion}

DAAs-based eradication of $\mathrm{HCV}$ is associated with improved glycemic control in patients with type 2 diabetes as evidenced by a significant reduction of mean FBG, Fasting insulin, HbA1c, and HOMA IR. There was a positive correlation between the treatment of $\mathrm{HCV}$ infection and the reduction of antidiabetic drugs used in the studied patients.

\section{References}

1. Ali A, Zein NN. Hepatitis C infection: A systemic disease with extrahepatic manifestations. Cleve Clin J Med. 
2005;72(11):1005-19.

2. Shintani Y, Fujie H, Miyoshi H, Tsutsumi T, Tsukamoto K, Kimura S, et al. Hepatitis C Virus Infection and Diabetes: Direct Involvement of the Virus in the Development of Insulin Resistance. Gastroenterology. 2004;126(3):8408.

3. Kawaguchi T, Ide T, Taniguchi E, Hirano E, Itou $\mathrm{M}$, Sumie S, et al. Clearance of HCV improves insulin resistance, beta-cell function, and hepatic expression of insulin receptor substrate 1 and 2 . Am J Gastroenterol. 2007;102(3):570-6.

4. Rui L. Energy metabolism in the liver. Compr Physiol. 2011;4(1):177-97.

5. Bohan EM. Diabetes mellitus and cirrhosis of the liver; a case report. Del Med J. 1947;19(11):212.

6. Kawaguchi T, Taniguchi E, Itou M, Sakata M, Sumie S, Sata M. Insulin resistance and chronic liver disease. World J Hepatol. 2011;3(5):99.

7. Gentric G, Maillet V, Paradis V, Couton D, L'Hermitte A, Panasyuk G, et al. Oxidative stress promotes pathologic polyploidization in nonalcoholic fatty liver disease. J Clin Invest. 2015;125(3):981-92.

8. TJ L, MG G. Only 20 years after the discovery of the Hepatitis C Virus (HCV), a cure is now likely for most people affected by this chronic infection, which carries a substantial disease burden, not only in the United States but also worldwide.1 The recent approval o. N Engl J Med. 2013;368(20):1907-17.

9. Razavi H, Waked I, Sarrazin C, Myers RP, Idilman $\mathrm{R}$, Calinas $\mathrm{F}$, et al. The present and future disease burden of hepatitis $\mathrm{C}$ virus (HCV) infection with today's treatment paradigm. J
Viral Hepat. 2014;21:34-59.

10. Falade-Nwulia O, Suarez-Cuervo C, Nelson DR, Fried MW, Segal JB, Sulkowski MS. Oral direct-acting agent therapy for hepatitis $\mathrm{c}$ virus infection: A systematic review. Ann Intern Med. 2017;166(9):637-48.

11. Nahon P, Bourcier V, Layese R, Audureau E, Cagnot C, Marcellin P, et al. Eradication of Hepatitis C Virus Infection in Patients With Cirrhosis Reduces Risk of Liver and Non-Liver Complications. Gastroenterology. 2017 Jan;152(1):142-156.e2.

12. Drazilova S, Gazda J, Janicko M, Jarcuska P. Chronic Hepatitis C Association with Diabetes Mellitus and Cardiovascular Risk in the Era of DAA Therapy. Can J Gastroenterol Hepatol. 2018;2018.

13. Pawlotsky J-M, Negro F, Aghemo A, Berenguer M, Dalgard O, Dusheiko G, et al. EASL recommendations on treatment of hepatitis C 2018. J Hepatol. 2018;69(2):461511.

14. Hashim AEM, Kandeel HT, Hendy OM, ElMola K, El-Raey FM, Attia MSM. Effect of new direct-acting antiviral drugs on insulin resistance and glycemic control after treatment of chronic hepatitis $\mathrm{C}$ virus infection in type 2 diabetic patients. Al-Azhar Assiut Med J. 2017;15(4):187.

15. Hum J, Jou JH, Green PK, Berry K, Lundblad $\mathrm{J}$, Hettinger BD, et al. Improvement in glycemic control of type 2 diabetes after successful treatment of hepatitis c virus. Diabetes Care. 2017;40(9):1173-80.

16. Ciancio A, Bosio R, Bo S, Pellegrini M, 
Sacco M, Vogliotti E, et al. Significant improvement of glycemic control in diabetic patients with $\mathrm{HCV}$ infection responding to direct-acting antiviral agents. J Med Virol. 2018;90(2):320-7.

17. Pashun RA, Shen NT, Jesudian A. Markedly Improved Glycemic Control in Poorly Controlled Type 2 Diabetes following Direct Acting Antiviral Treatment of Genotype 1
Hepatitis C. Case Reports Hepatol. 2016;2016:1-3.

18. Huang JF, Huang CF, Yeh ML, Dai CY, Hsieh MH, Yang JF, et al. The outcomes of glucose abnormalities in chronic hepatitis $\mathrm{C}$ patients receiving interferon-free direct antiviral agents. Kaohsiung J Med Sci. 2017;33(11):56771.

To cite this article: Ayman M. AlBadawy, Mohamed Shawky, Islam A. ElShazly, Rasha O. AbdelMoneim,. Impact of Hepatitis $C$ virus Infection and direct-acting Oral Antiviral Drugs on glycaemic state in Type 2 Diabetic Patients. BMFJ 2021;38(1):185-195. DOI: 10.21608/bmfj.2021.58139.1371 